\title{
THE DISCOVERY OF BIOFILM-FORMING PROTOZOA IN HUMAN BLOOD
}

\author{
Marian Simka \\ College of Applied Sciences, Department of Nursing, Ruda Śląska, Poland
}

EDITORIAL COMMENTARY

Phlebological Review 2014; 22, 1: 38-39

DOI: $10.5114 /$ pr.2014.46644
In this issue's paper Fry and colleagues have described protozoal microorganisms that they found in peripheral blood drawn from several patients suffering from chronic illnesses, such as: lupus erythematosus, chronic fatigue syndrome, fibromyalgia, multiple sclerosis, amyotrophic lateral sclerosis and arteriosclerosis [1]. Of note, these protozoa - although genetically related to already known organisms, primarily of aquatic origin - probably represented new species that have never been previously described. Moreover, they seemed to form biofilm communities in the bloodstream. Of course, this intriguing finding should be confirmed by independent researches, and protozoa described in this paper should be revealed in more patients (actually, in this preliminary report only a single case of each disease has been portrayed). Still, there are several potential breakthroughs in our understanding chronic incurable human diseases, providing that other scientists would also demonstrate such biofilm-forming protozoa in the patients' blood.

Firstly, bacterial and fungal biofilms are recognized important players in human pathology. In cases where the bacteria or fungi succeed in forming a biofilm, the infection often becomes difficult to treat and will develop into a chronic state. On the contrary, much less is known about protozoal biofilms. Some of them can form a biofilm in vitro [2], yet protozoal biofilm have not yet been described in the human blood.

Secondly, although protozoa described by Fry and colleagues can be of minor clinical relevance for their human hosts, a pathological role of these microorganisms cannot be ruled out. The strongest body of evidence supporting a hypothetical protozoal infection as a cause of the disease comes from the research on multiple sclerosis. Actually, the very idea that multiple sclerosis could be triggered by an infectious microorganism is not new and there are important data supporting the role for such a factor involved [3-6]. But - of as yet - not a single microorganism meeting the criteria of a causative factor has been identified. Until now, the researchers have primarily examined viruses. Others suggested a role for several bacterial species (e.g. Chlamydia pneumoniae) - similarly, with inconclusive results. What is new and promising in the Fry's and colleagues strategy is the focus at a different class of microorganisms, namely the protozoa. Potential pathological role of not yet identified protozoal parasite has already been suggested, since epidemiological and clinical features of multiple sclerosis point toward such microorganisms as a causal factor of the disease [7]. Yet, it should be remembered that standard tissue staining and other routine laboratory techniques may be inadequate to reveal many protozoa $[8,9]$. Thus, a search for protozoa using metagenomic essays and novel staining laboratory techniques seems very promising [10, 11]. In multiple sclerosis patient described in this paper, Fry and colleagues revealed a protozoan genetically related to Ochromonas sp., mixotrophic (thus, a parasitic species in this group should not be a surprise) unicellular golden algae. Of note, it is known that kindred protozoa: trypanosomes evoke autoimmune diseases in humans and animals through cross-reactivity between antigens of the parasite and those of the host $[12,13]$. It is commonly believed that multiple sclerosis develops as the result of autoimmune reaction against central nervous system myelin antigens, but a trigger of such an autoimmunity remains elusive. Interestingly, research has revealed myelin-like structures in Ochromonas danica cells [14]. It is tempting to speculate that in a case of infection by a similar protozoan, immune cross-reactivity between the myelin-like antigen derived from the protozoan and myelin proteins of the brain can develop, finally resulting in chronic neurologic autoimmune disease.

There is also another fact supporting a potential role for protozoa in the pathogenesis of multiple sclerosis. These patients present with high prevalence of structural abnormalities of the extracranial veins draining the central nervous system (the so-called chronic cerebrospinal venous insufficiency), usually adjacent to the junction of the internal jugular veins with the brachiocephalic veins. Of note, this is also the area of termination of the thoracic and right lymphatic ducts. Thibault has hypothesized that these anatomical relationships between the lymphatic and venous systems may allow a secondary spread of an infectious agent of multiple sclerosis through the lymphatic system and lead to infective phlebitis in the area of the jugular valve [15]. Although chronic cerebrospinal venous insufficiency in multiple sclerosis patients is usually discussed in terms of anatomical abnormalities of the jugular valves [16] an IVUS study has already described intraluminal "hyperechoic filling defects" in the area of the jugular valve in patients with multiple sclerosis [17]. 
Most likely, these structures were not thrombi, since no thrombi were detected by any of the venography studies. One explanation of this phenomenon could be that these artifacts represented separate regions of static blood [18]. But it could also be that actually these flow abnormalities resulted from biofilm streamers [19] formed by protozoa found by Fry and colleagues. Moreover, hypoxemic conditions in such a diseased vein may favor chronic protozoal infection through hypoxia-driven impairment of human immune cells [20].

Regarding other chronic diseases examined by the Authors, current evidence supporting potential role for protozoa in their pathogenesis is rather slim, yet such a role cannot be ruled out. Considering that all these chronic diseases are incurable and their cause is unclear (even in the case of arteriosclerosis), we await confirmation of the research released in this issue of Phlebological Review by other independent groups.

The author declares no conflict of interest.

\section{References}

1. Fry S.E., Ellis J.E., Shabilla M.A., Martinez D.L., Schwarz R., Heuser R., Moschonas C. Putative biofilm-forming organisms in the human vasculature: expanded case reports and review of the literature. Phlebol Rev 2014; 22: 24-37.

2. Oberholzer M., Lopez M.A., McLelland B.T., Hill K.L. Social motility in African trypanosomes. PLoS Pathogens 2010; 6: e1000739.

3. Kurtzke J.F. Epidemiology of multiple sclerosis. Does this really point toward an etiology. Lectio doctoralis. Neurol Sci 2000; 21 : 383-403.

4. Kurtzke J.F. Multiple sclerosis in time and space - geographic clues to cause. J Neurovirol 2000; 6 (Suppl. 2): S134-S140.

5. Murrell T.G., Harbige L.S., Robinson I.C. A review of the aetiology of multiple sclerosis: an ecological approach. Ann Hum Biol 1991; 18: 95-112.

6. Wallin M.T., Heltberg A., Kurtzke J.F. Multiple sclerosis in the Faroe Islands. 8. Notifiable diseases. Acta Neurol Scand 2010; 122: 102-109.

7. Simka M. What could be a primary cause of multiple sclerosis: Is it an autoimmunity triggered by chronic protozoan infection? J Neuroinfect Dis 2013; 4 ID: 235688; doi: 10.4303/jnd/235688.

8. Benvenuti L.A., Roggério A., Freitas H.F., Mansur A.J., Fiorelli A., Higuchi M.L. Chronic American trypanosomiasis: parasite persistence in endomyocardial biopsies is associated with highgrade myocarditis. Ann Trop Med Parasitol 2008; 102: 481-487.

9. Chimelli L. A morphological approach to the diagnosis of protozoal infections of the central nervous system. Patholog Res Int 2011; 2011: 290853.

10. Hyman R.W., St Onge R.P., Kim H., Tamaresis J.S., Miranda M., Aparicio A.M., Fukushima M., Pourmand N., Giudice L.C., Davis R.W. Molecular probe technology detects bacteria without culture. BMC Microbiol 2012; 12: 29.

11. Nazeer J.T., Khalifa K.S., von Thien H., El-Sibaei M.M., Abdel-Hamid M.Y., Tawfik R.A., Tannich E. Use of multiplex real-time PCR for detection of common diarrhea causing protozoan parasites in Egypt. Parasitol Res 2013; 112: 595-601.
12. Al-Sabbagh A., Garcia C.A., Diaz-Bardales B.M., Zaccarias C., Sakurada J.K., Santos L.M. Evidence for cross-reactivity between antigen derived from Trypanosoma cruzi and myelin basic protein in experimental Chagas disease. Exp Parasitol 1998; 89: 304-311.

13. Perty K., Voisin P., Baltz T., Labouesse J. Epitopes common to trypanosomes (T. cruzi, T. dionisii and T. vespertilionis (Schizotrypanum)): astrocytes and neurons. J Neuroimmunol 1987; 16: 237-252.

14. Aaronson S., Behrens U., Orner R., Haines T.H. Ultrastructure of intracellular and extracellular vesicles, membranes, and myelin figures produced by Ochromonas danica. J Ultrastruct Res 1971; 35: 6418-6430.

15. Thibault P.K. Multiple sclerosis: a chronic infective cerebrospinal venulitis. Phlebology 2012; 27: 207-218.

16. Simka M., Hubbard D., Siddiqui A.H., Dake M.D., Sclafani S.J., Al-Omari M., Eisele C.G., Haskal Z.J., Ludyga T., Miloševič Z.V., Sievert H., Stehling M.K., Zapf S., Zorc M.; International Society for Neurovascular Disease. .Catheter venography for the assessment of internal jugular veins and azygos vein: position statement by expert panel of the International Society for Neurovascular Disease. Vasa 2013; 42: 168-176.

17. Zivadinov R., Karmon Y., Dolic K., Hagemeier J, Marr K., Valnarov V., Kennedy C.L., Hojnacki D., Carl E.M., Hopkins L.N., Levy E.I., Weinstock-Guttman B., Siddiqui A.H. Multimodal noninvasive and invasive imaging of extracranial venous abnormalities indicative of CCSVI: results of the PREMiSe pilot study. BMC Neurol 2013; 13: 151.

18. Simka M. Chronic cerebrospinal venous insufficiency: current perspectives. J Vasc Diagn 2014; 2: 1-13.

19. Drescher K., Shen Y., Bassler B.L., Stone H.A. Biofilm streamers cause catastrophic disruption of flow with consequences for environmental and medical systems. PNAS 2013; 110: 43454350.

20. Mahnke A., Meier R.J., Schatz V., Hofmann J., Castiglione K., Schleicher U., Wolfbeis OS., Bogdan C., Jantsch J. Hypoxia in Leishmania major skin lesions impairs the NO-dependent leishmanicidal activity of macrophages. J Invest Dermatol 2014; 134: 2339-2346. 